\title{
THE
}

2015

\section{Automatic sleep apnea detection using fuzzy logic}

Aminollah Golrou

Keivan Maghooli

Amir Mohammad Amiri

University of Rhode Island

Kunal Mankodiya

University of Rhode Island, kunalm@uri.edu

Kazem Ghaemi

Follow this and additional works at: https://digitalcommons.uri.edu/ele_facpubs

The University of Rhode Island Faculty have made this article openly available.

Please let us know how Open Access to this research benefits you.

This is a pre-publication author manuscript of the final, published article.

Terms of Use

This article is made available under the terms and conditions applicable towards Open Access Policy Articles, as set forth in our Terms of Use.

\section{Citation/Publisher Attribution}

Golrou, A., Maghooli, K., Amiri, A. M., Mankodiya, K., \& Ghaemi, K. (2015, December 12). Automatic sleep apnea detection using fuzzy logic. 2015 IEEE Signal Processing in Medicine and Biology Symposium (SPMB). Philadelphia, PA, USA. doi: 10.1109/SPMB.2015.7405469

Available at: http://dx.doi.org/10.1109/SPMB.2015.7405469

This Conference Proceeding is brought to you for free and open access by the Department of Electrical, Computer, and Biomedical Engineering at DigitalCommons@URI. It has been accepted for inclusion in Department of Electrical, Computer, and Biomedical Engineering Faculty Publications by an authorized administrator of DigitalCommons@URI.For more information, please contact digitalcommons-group@uri.edu. 


\title{
Automatic Sleep Apnea Detection Using Fuzzy Logic*
}

\author{
Aminollah Golrou ${ }^{1}$, Keivan Maghooli ${ }^{1}$, Amir Mohammad Amiri ${ }^{2}$, Kunal Mankodiya ${ }^{2}$ and Kazem Ghaemi ${ }^{3}$
}

\begin{abstract}
The obstructive sleep apnea (OSA) is one of the most important sleep disorders characterized by obstruction of the respiratory tract and cessation in respiratory flow level. Currently, apnea diagnosis is mainly based on the Polysomnography (PSG) testing during sleeping hours, however, recording the entire signals during nights is a very costly, timeconsuming and difficult task. The goal of this study is to provide and validate an automatic algorithm to analyze four PSGrecordings and detect the occurrence of sleep apnea by noninvasive features. Four PSG signals were extracted from oxygen saturation (SaO2), Transitional air flow (Air Flow), abdominal movements during breathing (Abdomen mov.) and movements of the chest (Thoracic mov.). We describe a fuzzy algorithm to compensate the imprecise information about the range of signal loss, regarding the expert opinions. Signal classification is implemented minute-by-minute and for 30 labeled samples of MIT/BIH data sets (acquired from PhysioNet). The obtained data from 18 apnea subjects (11 males and 7 females, mean age 43 years) were categorized in three output signals of apnea, hypopnea and normal breathing. The proposed algorithm shows proficiency in diagnosing OSA with acceptable sensitivity and specificity, respectively $86 \%$ and $87 \%$.
\end{abstract}

\section{INTRODUCTION}

One of the most common sleep disorders is breathing interruptions also known as Sleep Apnea Syndrome (SAS). Repeated episodes of SAS interfere with normal sleeping pattern and reduce the sleeping quality. It must take at least 10 seconds to be considered sleep apnea and may occur 5 to 30 times in an hour of night sleep [1]. Some patients tolerate sleep apnea more than 300 times a night and this will consequently lead to daytime sleepiness, headaches, decreased quality of life and depression [2], [3]. Approximately, 3-7\% of male subgroups and 2-5\% of females in general adult categories suffer from SAS difficulties [4].

A direct relationship has been elucidated between OSA and cardiovascular diseases, but it is not clear that OSA is the main cause for greater prevalence of cardiac arrhythmia in these patients, or both of these obstacles are originated from a common factor such as obesity; since obesity and high blood pressure are the leading factors in cardiovascular diseases occurrence [3].

\footnotetext{
*This work was not supported by any organization

1 Aminollah Golrou and Keivan Maghooli are with Department of Biomedical Engineering, Tehran Science and Research Branch, Islamic Azad University, Tehran, Iran. amingolrou@bums.ac.ir, K_maghooliesrbiau.ac.ir

2 Amir Mohammad Amiri and Kunal Mankodiya are with the Department of Electrical, Computer, and Biomedical Engineering, University of Rhode Island, Kingston, RI 02881, USA. \{amiri, kunalm\}@ele.uri.edu

${ }^{3}$ Kazem Ghaemi is with Birjand University of Medical Science, Birjand, Iran. ghaemi@bums.ac.ir
}

Abnormal breathing mode is usually detected by airflow cessation during sleep; OSA is the obstruction in upper respiratory airway and Central Sleep Apnea (CSA) is caused by brain malfunction in controlling airflow and breathing system [5]. Commonly two apneic events occur which are described as follows: i) More than $75 \%$ reduction in respiratory flow signal for 10 seconds or more (apnea), ii) More than $45 \%$ for 10 seconds or more (Hypopnea) [6]. The latter form is followed by the reduction in oxygen saturation and is mainly diagnosed in male adults [7].

The basic method for detecting both types of disorders is performed by a full PSG recording test, afterward, identifying the events and constructing an apnea index on the basis of apneic events recorded during each sleeping hour [6]. Various signals such as electroencephalogram (EEG) and electrocardiogram (ECG), Electrocardiography (EOG), respiration flow, patient movements, and $\mathrm{SaO} 2$ are recorded to implement the test. This procedure helps the physician to understand if the patient has reached the desired depth of sleep and is usually undertaken in the laboratory environment [8].

EEG and $\mathrm{SaO} 2$ profiles have been investigated and compared in [9] and 91\% sensitivity, $83.3 \%$ specificity and $88.5 \%$ accuracy were achieved. EEG signal was also surveyed by an artificial neural network (ANN) algorithm [10] to find a solution for SAS. ECG signals (using time-frequency distributions and dynamic features) have been proposed as an accurate diagnostic tool for OSA [11]. In another study [12], it has been assessed in combination with $\mathrm{SaO} 2$ to evaluate sleep quality and $79.75 \%$ specificity, $85.89 \%$ sensitivity and high accuracy (up to $92.67 \%$ ) were achieved.

Sleeping in the lab and away from a normal situation cause discomfort, furthermore, the original test is invasive, especially EEG and ECG which require several electrodes attached to the patient's body to record the electrical activity. PSG technique is very costly and requires one-night admission in the sleep laboratory, continuous monitoring and professional personnel to test for appropriate diagnosis, therefore, new simplified methods for monitoring and diagnosis of OSA are in demand. Sleep-wake state and oxygen saturation indexes resulted from unattached home PSG testing are in a close correlation with data obtained in the attended laboratory setting; in this context the discomfort scale of testing procedure will decline and, therefore, the accuracy increase [13], [14].

In this paper, we develop and validate an efficient fuzzylogic-based algorithm to detect breathing abnormalities using only four PSG signals. $\mathrm{SaO} 2$ and body movement are the main indicators in this survey and the main goal is to reduce 
the recording expenses and discomfort without accuracy reduction as shown in figure 1 .

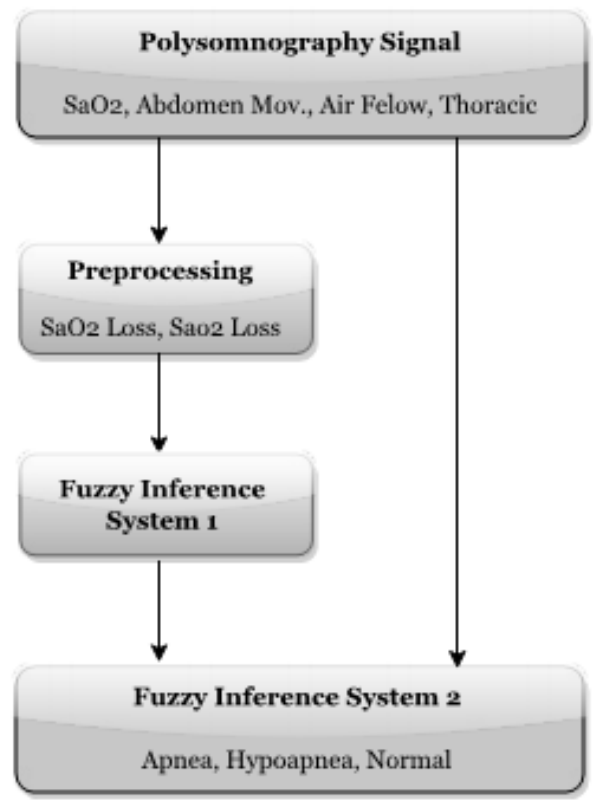

Fig. 1. The procedure of an automatic sleep apnea detection using fuzzy logic.

\section{METHOD}

This study presents relatively simple methods/tools based on fuzzy theory to detect the occurrence of OSA. For this purpose, only four signals; $\mathrm{SaO} 2$, Transitional air flow (AirFlow), Abdominal movements during breathing (Abdomen mov.) and Movements of the chest (Thoracic mov.) derived from registered PSG recordings were used (See figure 1).

First, the $\mathrm{SaO} 2$ signal search by two characteristics: signal loss and its duration. These amounts use as inputs in Fuzzy Inference System (FIS) which are based on the rules that defined for this system. For instance, the different sectors will be differentiated and regions of low $\mathrm{SaO} 2$ (desaturation) that are achieved. At each point where $\mathrm{SaO} 2$ detect lower than the expected value, the system starts to search for a specified period of time (30 seconds before the event) where three other signals will be scrutinized. The latter step, another fuzzy system scans three signals, if the signal level is reduced (related to the maximum value) in a common part of the signal and this reduction is greater than a specified threshold, the apnea occurs.

\section{A. Data Acquisition}

About 18 study data were derived from the apnea database corresponding to the MIT / BIH database. Air Flow, SaO2, Abdominal and Thoracic Movements were obtained from a full PSG record. Each record includes a period of about eight hours, and signal annotation is done minute-by-minute. A minute is labeled as an apnea case if it contains at least one condition of apnea or hypopnea, otherwise labeled as a non-apnea. The patients who his/her age and weight are between 27 to 63 years (4810.8) and 53 to $135 \mathrm{~kg}(86.322 .2)$, respectively. PhysioNet data are classified in two categories: Apnea Group (Class A, more than 100 minutes of apnea, mean age: 50 years in a range of 29 to 63 years) and, Normal Group (class C, less than 5 minutes of apnea, mean age of 33 years in a range of 27 to 64 years). Totally, 14,708 minutes of healthy and apnea cases have been used for training and testing. After receiving the signal, the sampling frequency is defined, and the initial preprocessing is applied to $\mathrm{SaO} 2$ (Figure 2 shows the signals obtained).
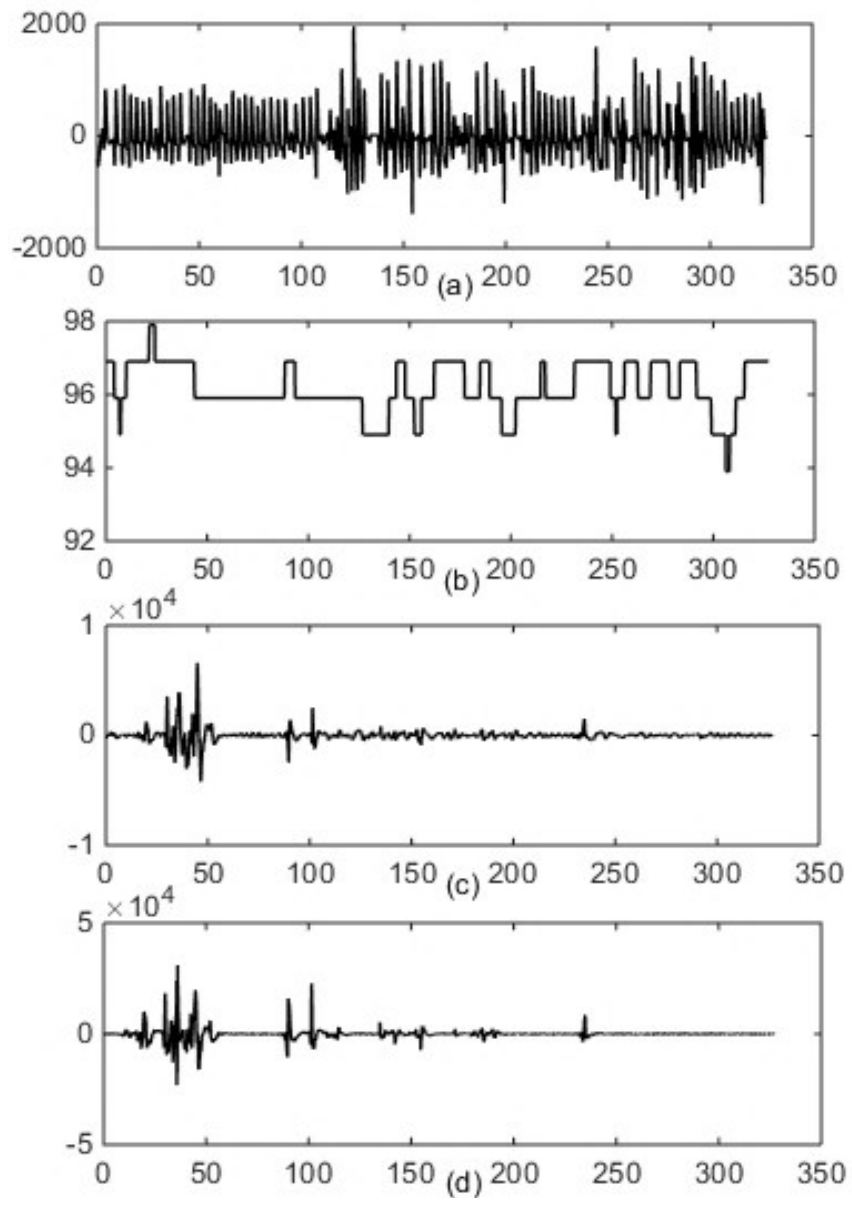

Fig. 2. The four main signals; a) airflow, b) $\mathrm{SpO}$ 2, c)Abdominal and d) Thoracic movments from PSG recordings after the initial pre-processing actions.

\section{B. Implementation}

Fuzzy systems approve or disapprove a word or a phrase based on relativity. This means instead of totally rejecting or accepting words, they are given a relativity to become acceptable or reject-able. These systems translate environmental issues to fuzzy systems (fuzzifying) and then using the rules defined by an expert a decision is made and system outputs are de- fuzzified depending on extracted rules.

In this study, first the oxygen saturation signal is considered and four signals are entered into MATLAB environment, the sampling frequency is defined to perform initial preprocessing. This pre-processing is applied to $\mathrm{SaO} 2$ signal, 
consequently the $\mathrm{SaO} 2$ signal loss and its duration are mined and the loss points are determined.

The signal loss points are extracted depending on the amount and the duration of signal loss and entered to fuzzy systems; the result of the fuzzy system and the outputs are considered as a criterion to detect apnea (signal loss in respiratory flow: more than $75 \%$ for 10 seconds or more) or hypopnea (signal loss in respiratory flow: more than $45 \%$ for 10 seconds or more). Then the first fuzzy system started searching the suspicious point to exhibit $\mathrm{SaO} 2$ signal loss and the results (basically the rules) are extracted from this system (See figure 3 and 4).

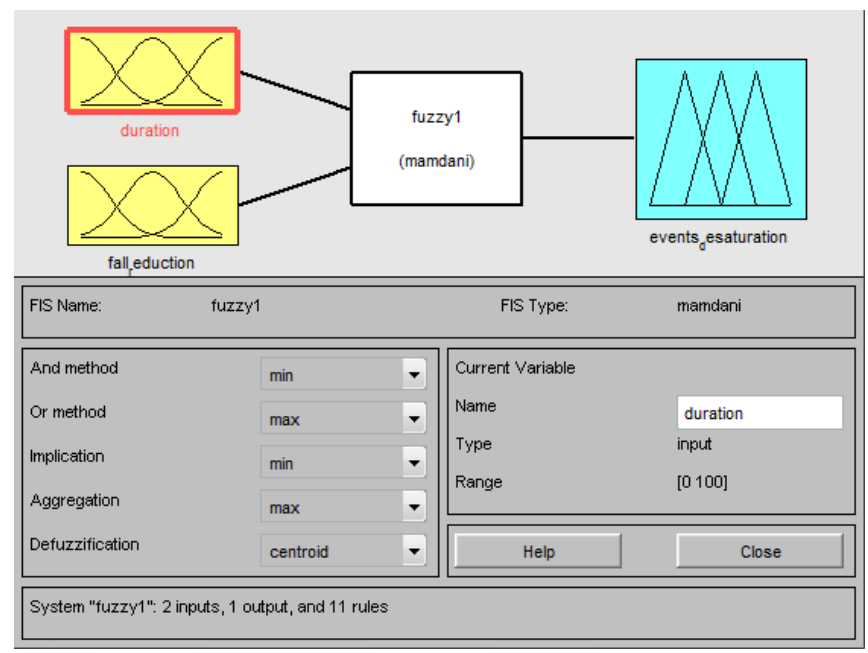

Fig. 3. Fuzzy System used in the first part of the project to identify suspicious points to $\mathrm{SaO} 2$ signal loss.

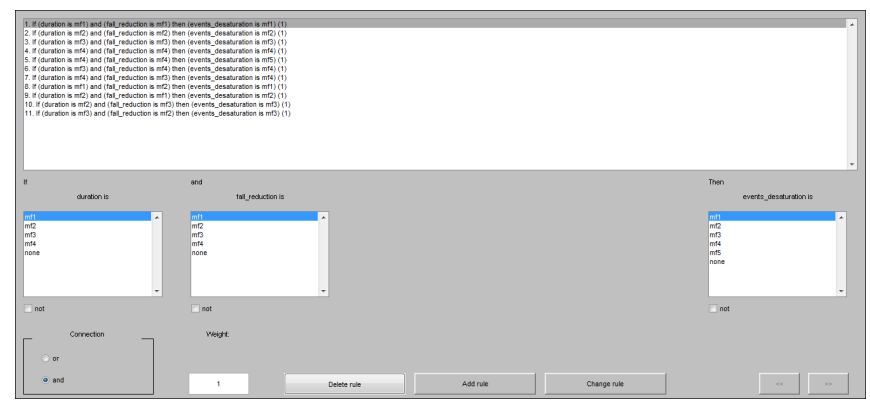

Fig. 4. The rules of the fuzzy system used in the first part of the project to detect suspicious points to Sao2 signal loss.

A 30 seconds region is defined on an interval before the $\mathrm{SaO} 2$ signal loss where a strong signal loss is detected due to maximum, and RU are extracted. Different factors were considered such as the amount of loss occurred before the first 30 seconds or the interval between two consecutive losses have to be less than 30 seconds.

Airflow signals, abdomen, and chest movements are scrutinized in 30 seconds before oxygen loss or searching space, and if the amount of losing is less than $40 \%$ of the maximum, and another hand the duration is more than 7 seconds, it is also suspected as apnea, and will be separated from signals. After calculating each signal loss duration, suspected points are extracted. When there is an interval overlap or repeated overlaps, the smaller intervals are considered, and duplications will be eliminated. The inputs of the fuzzy system are oxygen saturation, the duration and amount of signal loss obtained from the previous step. Subsequently, the detection results of apnea events are archived at three groups: normal breathing, apnea, and hypopnea which are shown in figure 5 and 6.

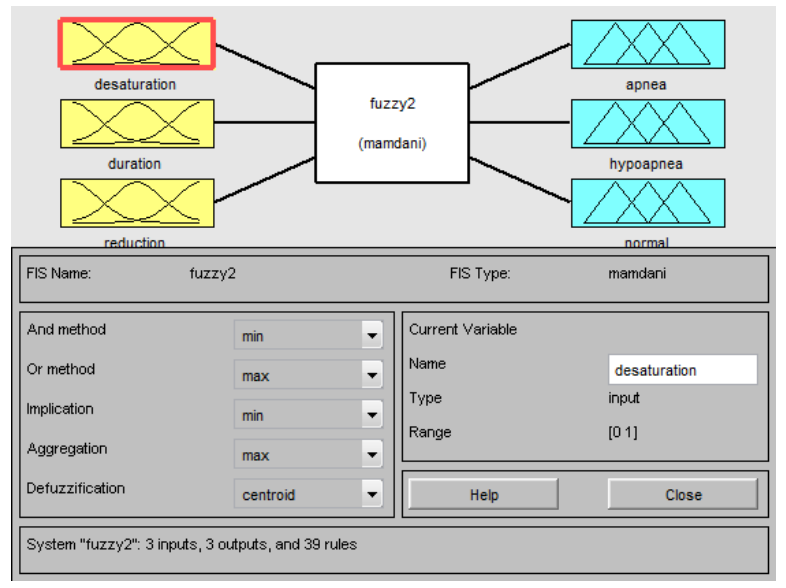

Fig. 5. Fuzzy System (FIS) used in the second part of the project to detect apnea, hypopnea or normal cases

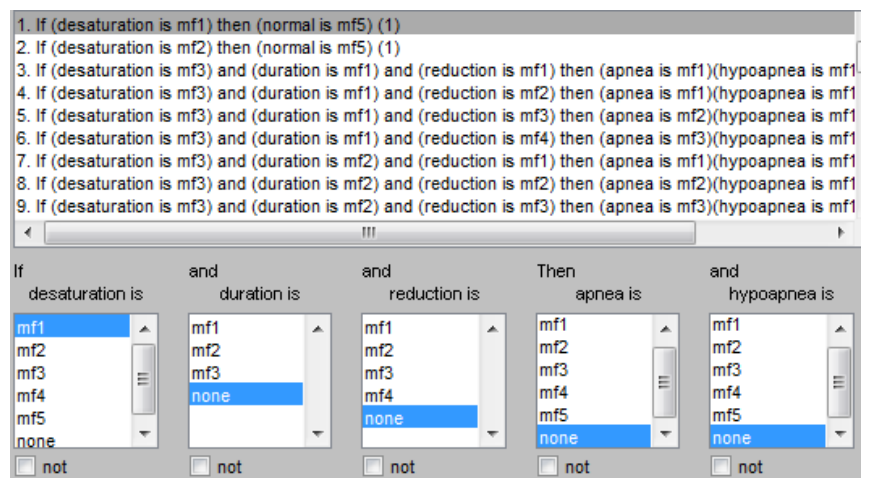

Fig. 6. The rules given to the fuzzy system (FIS), used in the second part of the project to detect apnea, hypopnea or normal cases.

\section{EXPERIMENTAL AND RESUlts}

To review and validate the proposed system in this paper, MIT / BIH polysomnography database is derived from PhysioNet and the signals are specifically labeled by experts. The results of this system are compared with 18 patients? PSG data and totally 14,708 minutes of healthy and apnea signals are utilized to test the system. Totally, 8325 apnea and hypopnea events are labeled by an expert.

As mentioned in the previous section, two fuzzy systems (FIS) are used in this research; In the first part, output (figure 7) is illustrated to detect time and $\mathrm{SaO} 2$ signal loss for a typical signal. As an instant, in part 12 and 16, the membership function is zero where the signal loss occurs in specific time duration (See figure 8). 


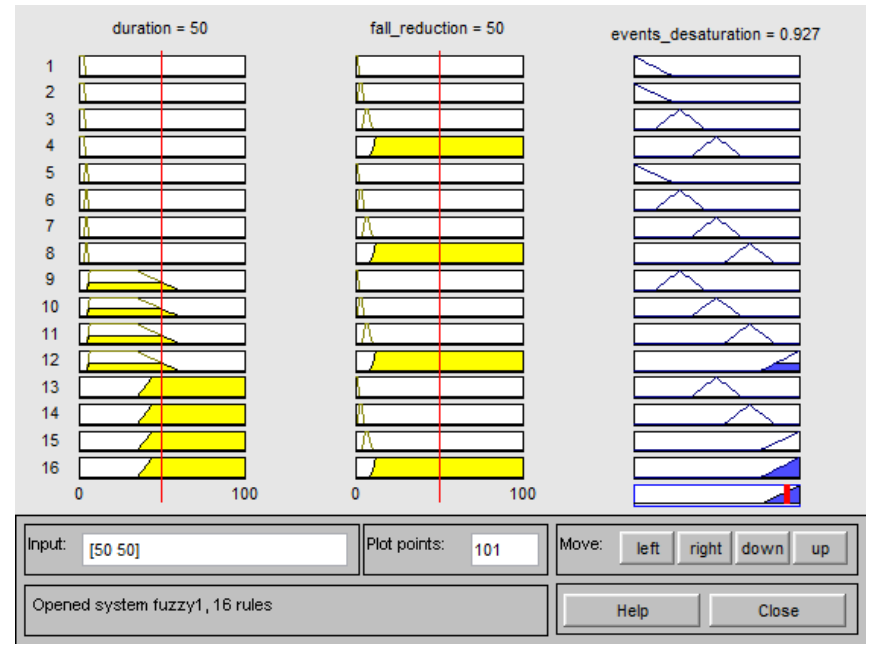

Fig. 7. Output Sample of the fuzzy system used in the first part.

In the second part, the apnea, hypopnea and normal signals are achieved using output of the first stage and searching the signals according to the rules of the second stage; in other words, the membership function of each signal can be calculated according to these functions.

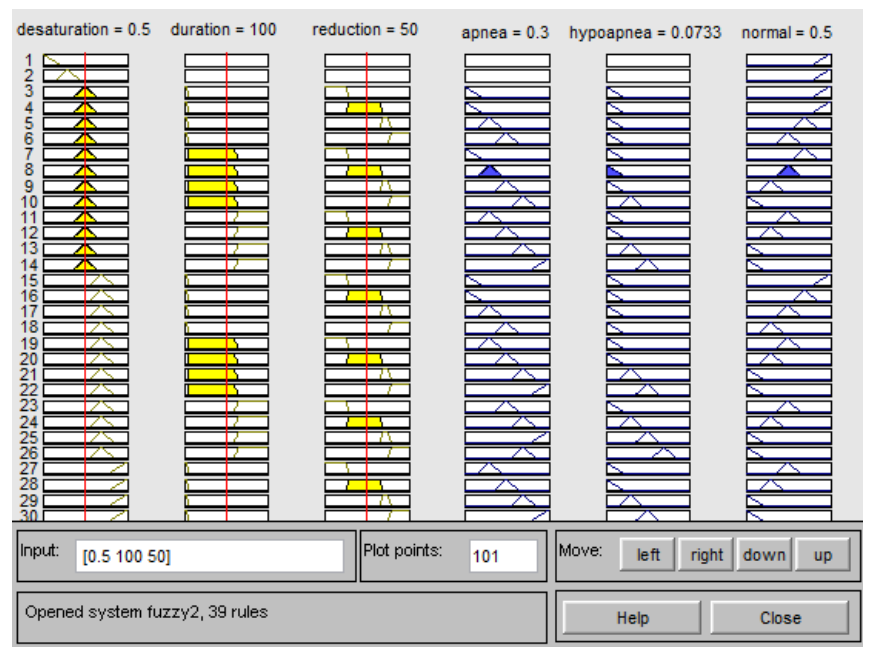

Fig. 8. Output sample of the fuzzy system rules used in the second part of the project (membership functions for the three forms of apnea, hypopnea and normal cases are calculated separately).

The results verify the validity and performance of the proposed algorithm with $83.7 \%$ accuracy, $86.3 \%$ sensitivity, and specificity $87.8 \%$. Table I shows the results in comparison with other works on PSG extracted signals. This is to certify that our fuzzy based algorithm has achieved acceptable performance.

\section{Discussion}

Chronic SAS are not well understood or recognized by primary care physicians and unfortunately, a small fraction of the cases has ever been diagnosed and treated. Previous methods to predict, detect and cure SAS are frequently based on PSG testing. Different biomedical features are extracted to survey sleep disorders. EEG and ECG have been reported in different studies [11] and to reduce the test discomfort and timely processing, short duration epochs of ECG data were extracted and evaluated [15]. Oxygen saturation obtained from pulse oximetry reported approximately $93.3 \%$ accuracy [16] and is a reliable criterion.

In this research, we investigated the possibility of SAS diagnosing using four PSG features: i) Blood oxygen saturation $(\mathrm{SaO} 2)$, ii) transitional airflow (Air Flow), iii) abdominal movements during breathing (Abdomen mov.) and iv) movements of the chest (Thoracic mov.). To perform this study, a fuzzy based algorithm was applied and demonstrated a high performance and accuracy.

As mentioned in implantation section, each of considered epochs includes duration of 30 seconds. Output results of the system have been studied from the perspective of the occurrence location and the type of event (apnea or hypopnea). The results are shown in Table II which obtained from the system are compared with 18 patient's PSG data, and a total of 14,708-minute apnea and healthy signal are used to test the system.

Totally, 8325 events are labeled as apnea and hypopnea events by an expert. Regarding the results the proposed algorithm shows $86 \%$ sensitivity and $88 \%$ specificity for detecting the occurrence of these events (for a total time of the 14,708 minutes signal); based on these results the system performance to detect and diagnose apnea and hypopnea are well described.

In this work, the results are obtained from the outputs of a fuzzy inference system based on a combination of rules taken from Mamdany [6]. We suggest to use other combinations such as Gdel in future studies and to compare the results with different combinations.

\section{CONCLUSion}

In this study, a fuzzy inference system has been suggested as a smart algorithm to detect the occurrence of sleep apnea and hypopnea. The advantages of such compatible and self-organize fuzzy systems can help facilitate people with diseases such as apnea, including continuous positive pressure system (CPAP).

In the proposed system, only four signals extracted from the full registered PSG recordings have been used. This can definitely lead to computational simplicity and lower cost than using the full data. Oxygen saturation and patient movements, the extracted features in this study, do not interfere with sleeping condition and reduce PSG discomfort. Another advantage of the proposed method is insensitive to the initial pre-processing and no need for selecting noise-free signals.

\section{ACKNOWLEDGMENT}

The research group is willing to thank Dr. Ali Sheikhani for his advantages consultancy. who is with Department of Biomedical Engineering, Tehran Science and Research Branch, Islamic Azad University, Tehran, Iran. 
TABLE I

COMPARISON BETWEEN THE EXTRACTED FEATURES AND RESULTED ACCURACY.

\begin{tabular}{|l|l|l|l|l|l|}
\hline Methods & Ref. & Extracted Features & Sen. & Spec. & Acc. \\
\hline Quiceno-Manrique et al. & 11 & ECG signal & NA & NA & 92.67 \\
\hline Alvarez et al. & 9 & SaO2 and EEG signal & 91 & 83.3 & 88.5 \\
\hline Almazaydeh et al. & 1 & Respiratoy signal & NA & NA & 80 \\
\hline Schrader et al. & 5 & ECG signal & 90.8 & 92.7 & NA \\
\hline Xie et al. & 12 & Sao2 and ECG & 79.75 & 85.89 & 84.40 \\
\hline Philip de Chazal et al. & 3 & ECG using linear discriminant analysis & 89 & 93 & NA \\
\hline Current Research & - & SaO2, Thoracic, Abdominal and Airflow & 86.3 & 87.8 & 87.3 \\
\hline
\end{tabular}

TABLE II

SYSTEM PERFORMANCE CRITERIA IN EXPERT OPINION TP $=$ TRUE

POSITIVE, TN = TRUE NEGATIVE, FP= FALSE POSITIVE, FN = FALSE

NEGATIVE, SENS $=$ SENSITIVITY, SPEC $=$ SPECIFICITY.

\begin{tabular}{|l|l|l|l|l|}
\hline Signal & Slp03 & Slp04 & Slp14 & Slp16 \\
\hline Epochs & 1200 & 1140 & 1320 & 1318 \\
\hline Apneic events (Expret) & 406 & 283 & 636 & 567 \\
\hline Apneic events (System) & 401 & 375 & 678 & 685 \\
\hline TP & 358 & 230 & 575 & 498 \\
\hline TN & 751 & 712 & 581 & 633 \\
\hline FP & 43 & 145 & 103 & 118 \\
\hline FN & 48 & 53 & 61 & 69 \\
\hline Sens. & 0.88 & 0.81 & 0.90 & 0.86 \\
\hline Spec. & 0.95 & 0.83 & 0.85 & 0.84 \\
\hline
\end{tabular}

\section{REFERENCES}

[1] L. Almazaydeh, K. Elleithy, M. Faezipour, and A. Abushakra, A. . Apnea Detection Based on Respiratory Signal Classification. Procedia Computer Science, 21, 2013, pp.310-316.

[2] S. Surani, et al. "Anxiety and Depression in Obstructive Sleep Apnea: Prevalence and Gender/Ethnic Variance." Current Respiratory Medicine Reviews 9.4, 2013, pp. 274-279.

[3] P. De Chazal, and C. Heneghan. "Apparatus for detecting sleep apnea using electrocardiogram signals." U.S. Patent No. 7,025,729. 11 Apr. 2006.

[4] N. M. Punjabi, "The epidemiology of adult obstructive sleep apnea." Proceedings of the American Thoracic Society 5.2, 2008, pp.136-143.

[5] M. Schrader, et al. "Detection of sleep apnea in single channel ECGs from the PhysioNet data base." Computers in Cardiology 2000. IEEE, 2000.

[6] D. lvarez-Estvez, and M. B. Vicente "Fuzzy reasoning used to detect apneic events in the sleep apnea-hypopnea syndrome." Expert Systems with Applications 36.4, 2009, pp. 7778-7785.

[7] A. J. Block, P.G. Boysen, J. W. Wynne and L. A. Hunt, "Sleep apnea, hypopnea and oxygen desaturation in normal subjects: a strong male predominance." New England Journal of Medicine 300.10, 1979, pp.513-517.

[8] Sze, W. J., P. Habibi, and H. Brooks. "G88 HISTORY and Clinical assessment versus Polysomnography for diagnosis of obstructive sleep apnoea?." Archives of Disease in Childhood 99.Suppl 1, 2014 pp. A37A38.

[9] D. Alvarez, R. Hornero, J. V. Marcos, F. Del Campo and M. Lopez, "Spectral analysis of electroencephalogram and oximetric signals in obstructive sleep apnea diagnosis." Engineering in Medicine and Biology Society, 2009. EMBC 2009. Annual International Conference of the IEEE. IEEE, 2009.

[10] R. LIN, R. G. LEE, C. L. TSENG, H. K. ZHOU, C. F. CHAO and J.A JIANG, "A new approach for identifying sleep apnea syndrome using wavelet transform and neural networks." Biomedical Engineering: Applications, Basis and Communications 18.03, 2006, pp. 138-143.

[11] A. F. Quiceno-Manrique and J. B. Alonso-Hernandez,'Detection of obstructive sleep apnea in ECG recordings using time-frequency distributions and dynamic features." Engineering in Medicine and Biology Society, 2009. EMBC 2009. Annual International Conference of the IEEE. IEEE, 2009.
[12] B. Xie and H. Minn, "Real-time sleep apnea detection by classifier combination." Information Technology in Biomedicine, IEEE Transactions on 16.3, 2012, pp. 469-477.

[13] WW. Flemons, M. R. Littner, J. A. Rowley, P. Gay., W. M. Anderson, D. W. Hudgel, ... and D.I Loube, "Home diagnosis of sleep apnea: a systematic review of the literature: an evidence review cosponsored by the American Academy of Sleep Medicine, the American College of Chest Physicians, and the American Thoracic Society." CHEST Journal 124.4, 2003, pp. 1543-1579.

[14] D. Zou, L. Grote, Y. Peker, U. Lindblad and J. Hedner, "Validation a portable monitoring device for sleep apnea diagnosis in a population based cohort using synchronized home polysomnography." SLEEPNEW YORK THEN WESTCHESTER- 29.3, 2006 pp. 367.372.

[15] L. Almazaydeh, E. Khaled, and M.Faezipour. "Obstructive sleep apnea detection using SVM-based classification of ECG signal features." Engineering in Medicine and Biology Society (EMBC), 2012 Annual International Conference of the IEEE. IEEE, 2012.

[16] L. Almazaydeh, M. Faezipour, and E. Khaled. "A Neural network system for detection of obstructive sleep apnea through $\mathrm{SpO} 2$ signal features." (2012). 